\title{
Opinión del alumnado sobre el perfil de ingreso del médico veterinario y zootecnista del Centro Universitario de los Altos (CUALTOS)
}

Opinion of students on the admission profile of the Veterinarian Zootechnician of Los Altos University Center (CUALTOS) Opinião dos alunos sobre o perfil de renda do cientista veterinário e animal no Centro Universitário de Altos (CUALTOS)

DOI: http://dx.doi.org/10.23913/ricsh.v6i11.116

Adriana de la Rosa Figueroa Universidad de Guadalajara, México mvzadelarossa@gmail.com

Alberto Esparza González Universidad de Guadalajara, México alberto.esparza@hotmail.com

\section{Resumen}

Este trabajo tiene como objetivo principal explicar algunas de las características personales de los alumnos que ingresan a la licenciatura en Medicina Veterinaria y Zootecnia del Centro Universitario de los Altos. La información se obtuvo mediante una investigación cualitativa y la aplicación de un examen de diagnóstico al $100 \%$ de los alumnos (42) de nuevo ingreso a la licenciatura en el calendario 2016-B. Para ello se contabilizaron el número de respuestas con resultado positivo en el examen de diagnóstico realizado al comienzo del semestre. De forma general, $51 \%$ de los alumnos tiene una noción básica sobre el perfil de ingreso de su carrera.

Palabras clave: perfil de ingreso, Medicina Veterinaria, características de ingreso, diagnóstico. 


\section{Abstract}

This work aims to explain some of the personal characteristics of the students admitted to the Bachelor's degree in Veterinarian Zootechnician of Los Altos University Center. Information was obtained through a qualitative research and the application of a diagnostic test to $100 \%$ of the students (42) first semester freshman into the degree course in the 2016B calendar. So were the number of responses with a positive result in the screening carried out at the beginning of the semester. In general, $51 \%$ of students have a basic notion of career entry profile.

Key words: admission profile, veterinary medicine, admission, characteristics, career entry profile, diagnosis.

\section{Resumo}

O principal objetivo deste artigo é explicar algumas das características pessoais dos alunos que entram licenciatura em Medicina Veterinária e Zootecnia da Centro de Medicina da Universidade de Los Altos. A informação foi obtida por meio de uma pesquisa qualitativa e a aplicação de um teste de diagnóstico de $100 \%$ dos estudantes (42) recém-admitidos para o grau no calendário 2016-B. Para isso, o número de respostas com um resultado positivo no teste de diagnóstico feito no início do semestre foram registrados. Geralmente, $51 \%$ dos alunos têm uma compreensão básica sobre o perfil de renda de sua carreira.

Palavras-chave: perfil admissão, Medicina Veterinária, características de renda, diagnóstico.

Fecha Recepción: Junio $2016 \quad$ Fecha Aceptación: Diciembre 2016 


\section{Introducción}

\section{Antecedentes}

Durante el siglo pasado, la medicina veterinaria en México se consideraba como una disciplina agregada a la agronomía. La carrera de agronomía trataba de la producción de alimentos, incluyendo aspectos zootécnicos, mientras que los veterinarios se dedicaban casi exclusivamente al tratamiento de las enfermedades de los caballos, el medio de locomoción de la época. El Colegio Nacional de Agricultura y Veterinaria inició los cursos para veterinarios en 1856, con un plan de estudios de cuatro años en el que se impartían química, zoología, anatomía, equitación, inglés, alemán, patología, clínica hipiátrica y cirugía. Los cinco egresados entre 1861 y 1869 ingresaron al Cuerpo de Caballería del Ejército.

En 1870, Benito Juárez encargó a Gabino Barreda la organización del Sistema Educativo Mexicano, basado en principios positivistas que consistían en:

a) Extender lo más posible los beneficios de la educación; y

b) Generalizar el conocimiento de las ciencias exactas y naturales.

Como un resultado de esta política se fundó la Escuela Nacional de Agricultura y Veterinaria de San Jacinto, cuyo objetivo era formar administradores de fincas, con un plan de estudios de cuatro años en el que se refleja la importancia de las asignaturas como las matemáticas, la física y la química, incluidas por la fuerte influencia del positivismo en la educación. En 1883, se inició el cambio del concepto del médico veterinario como hipiatra al de médico veterinario de todas las especies domésticas; nacen así las asignaturas de anatomía comparada, fisiología comparada y se introducen además la zootecnia general, la medicina legal y la jurisprudencia veterinaria en el último año de la carrera.

Los años de los movimientos armados trajeron consigo un estancamiento de décadas en todos los sectores de la educación. Hasta 1925 ocurrió un cambio muy importante en la sociedad que se reflejó intensamente en la educación veterinaria (Educación Veterinaria en México, 2015). 
El perfil de ingreso a la licenciatura de Medicina Veterinaria y Zootecnia, que describe el Centro Universitario de los Altos, de la Universidad de Guadalajara, menciona que los requisitos que debe cumplir un aspirante para el ingreso a esta licenciatura deben ser:

1) Actitudes (comportamiento y disposiciones). Las características particulares del desarrollo personal que cada individuo ha aprehendido y madurado como producto de su experiencia de vida deben ser: iniciativa, responsabilidad, autoaprendizaje, disciplina, liderazgo, sociabilidad, honestidad, ética, tenacidad, vocación de servicio, afinidad e interés por los fenómenos biológicos, disponibilidad para el trabajo de laboratorio y de campo.

2) Aptitudes (capacidades y habilidades). Disposición natural que la persona posee o adquiere, son: saber aprovechar los recursos disponibles; saber dirigir; capacidad para administrar, realizar trabajo en equipo y para el análisis clínico; capacidad de síntesis y de abstracción; pensamiento lógico matemático; habilidad en la lectura de comprensión, en redacción y composición, creatividad; habilidad en comunicación oral y escrita.

3) Antecedentes académicos. Son los estudios formales realizados previamente. Haber cursado el bachillerato (CUALTOS, 2016).

El principal objetivo de este estudio es conocer las opiniones de los alumnos de primer ingreso a la licenciatura en Medicina Veterinaria y Zootecnia que fueron admitidos durante el ciclo escolar 2016 B, en el Centro Universitario de los Altos (CUALTOS), perteneciente a la Benemérita Universidad de Guadalajara.

\section{Material y métodos}

Se aplicó un cuestionario diagnóstico al $100 \%$ de los alumnos de primer ingreso del calendario 2016 A del Centro Universitario de los Altos, para conocer los lineamientos básicos que se tienen sobre el perfil de ingreso del Médico Veterinario y Zootecnista en México. Este cuestionario buscó revelar, principalmente, el conocimiento, la relación directa que tienen los estudiantes con la producción animal, si tienen algún ingreso o negocio comercial relacionado con el medio pecuario, y el pensamiento que ellos tienen directamente de la licenciatura y del CUALTOS. 
Se realizó, además, una investigación documental, comparativa y descriptiva sobre los diferentes perfiles de ingreso de las principales 15 universidades mexicanas que imparten este plan de estudios, para conocer las características básicas y competencias que debe desarrollar cualquier estudiante.

\section{Resultados}

Con base en los resultados del examen de diagnóstico aplicado a los alumnos de primer ingreso a la licenciatura de Medicina Veterinaria y Zootecnia, se puede constatar que solamente $51 \%$ de estos alumnos afirma conocer el perfil de egreso de M.V.Z. del Centro Universitario de los Altos.

Figura 1. Conocimiento sobre el perfil de ingreso.

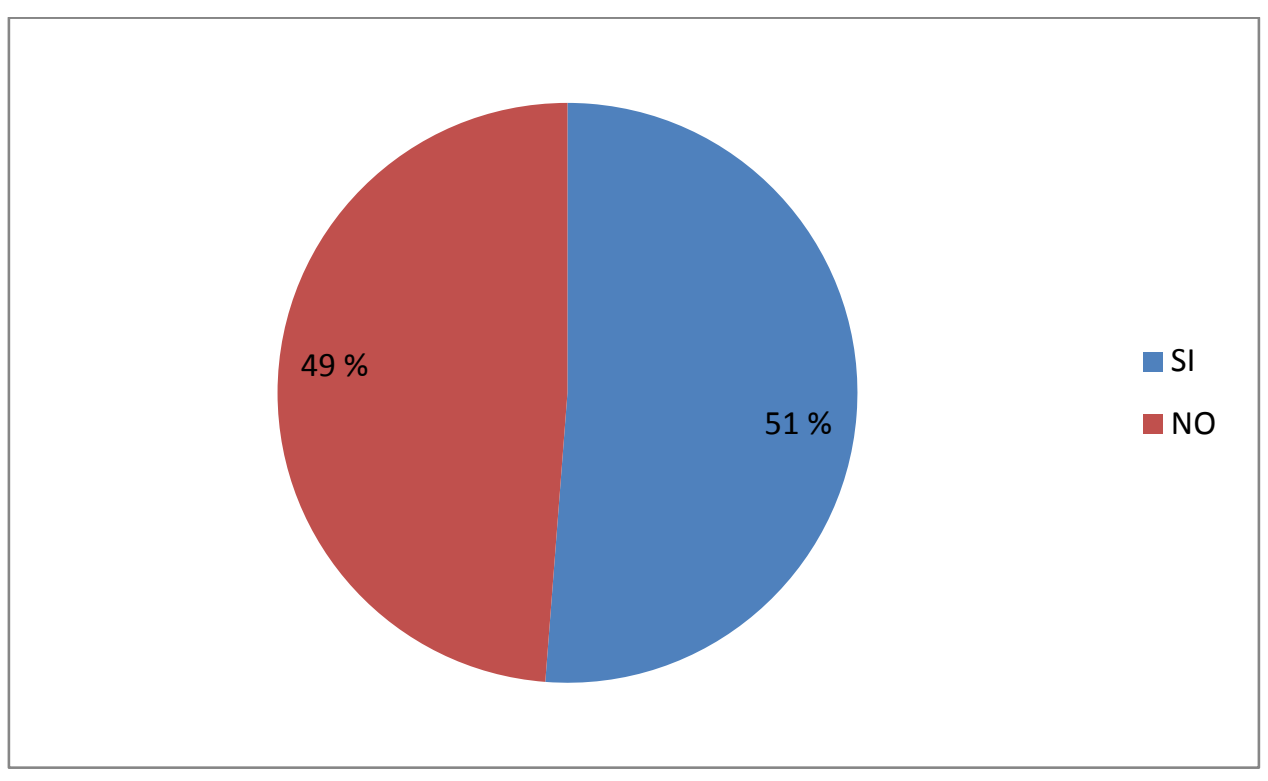

A la pregunta ¿tiene usted relación directa con la producción animal de tal forma que su desarrollo profesional esté ligado a su vida personal?, 54 \% contestó afirmativamente. 
Figura 2. Relación directa con la producción animal.

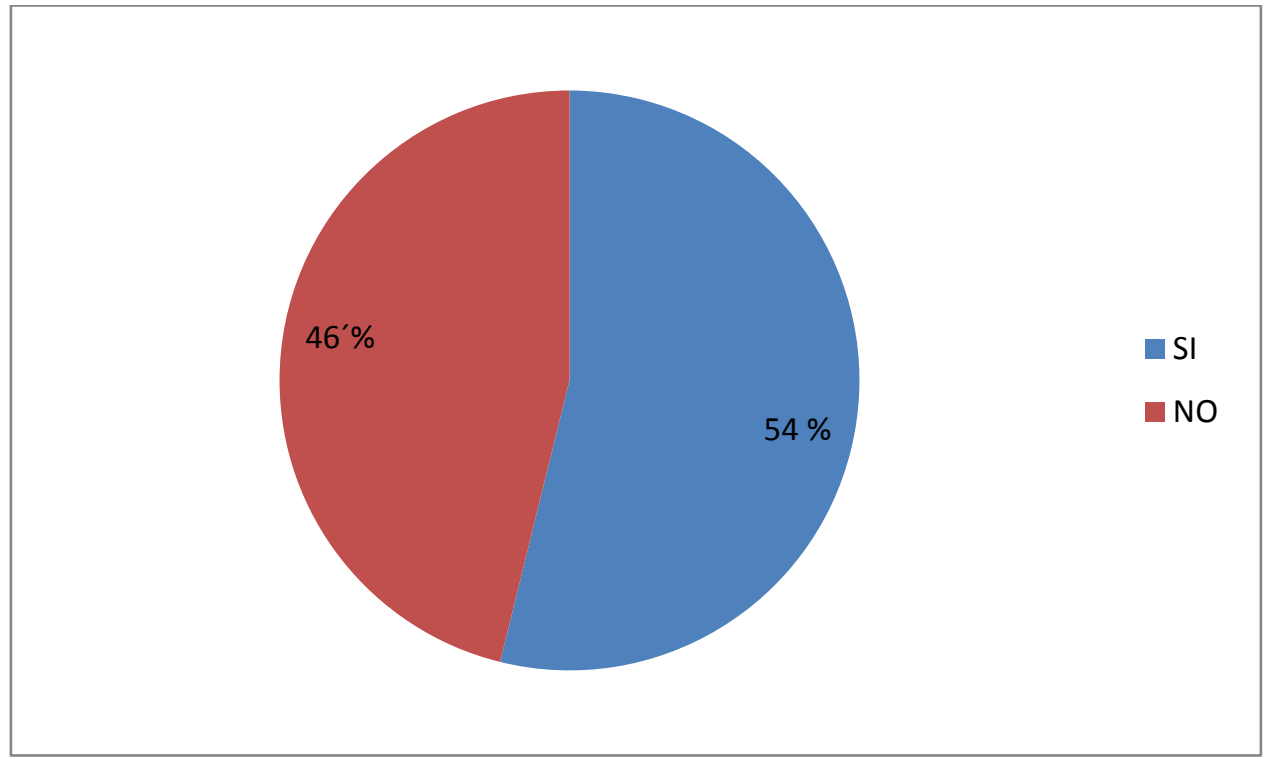

Al preguntar ¿cuáles son las especies animales que ha manejado durante los últimos cinco años en caso de tener una fuente de ingresos de origen pecuaria?, la respuesta de los alumnos fue la siguiente:

Figura 3. Fuente de ingreso económica de origen pecuario.

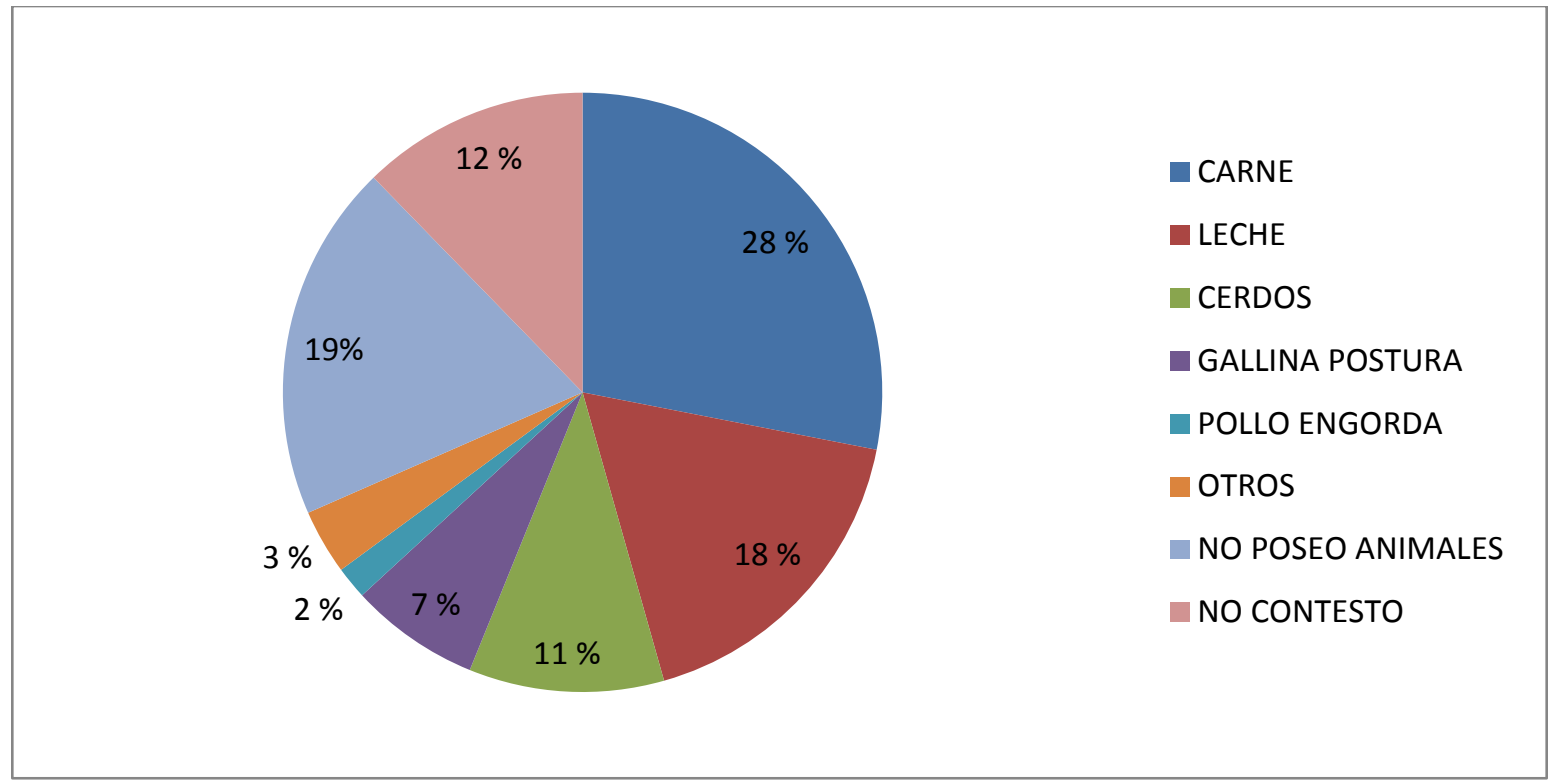

¿Su familia tiene o ha tenido algún negocio comercial relacionado con animales? $54 \%$ contestó afirmativamente: 
Figura 4. Negocio comercial familiar relacionado con animales.

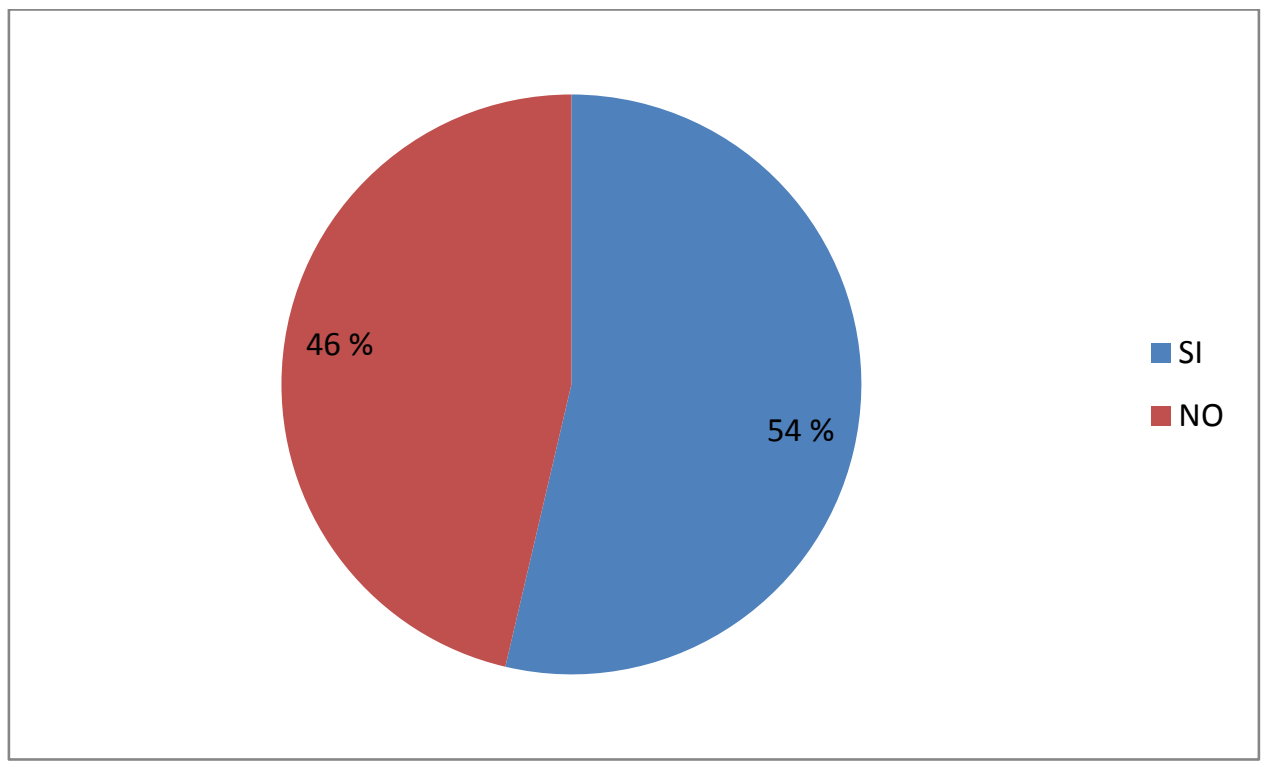

La siguiente pregunta fue: ¿qué espera de su formación como médico veterinario y zoootecnista? con las opciones de respuesta:

a) Que mejore su calidad de vida.

b) Que pueda aplicar sus conocimientos en su medio particular (granja, rancho, establo, otro).

c) Que pueda ser contratado por una buena organización.

d) Que pueda usted generar su autoempleo como empresario.

Las respuestas mostraron las variantes mostradas a continuación:
A. Que mejore su calidad de vida
B. Que pueda aplicar sus conocimientos en su medio particular (granja, rancho, establo, otro)
C. Que pueda ser contratado por una buena organización
D. Que pueda Usted generar su autoempleo como Empresario 
Figura 5. ¿Qué espera un alumno del CUALTOS de su formación veterinaria?

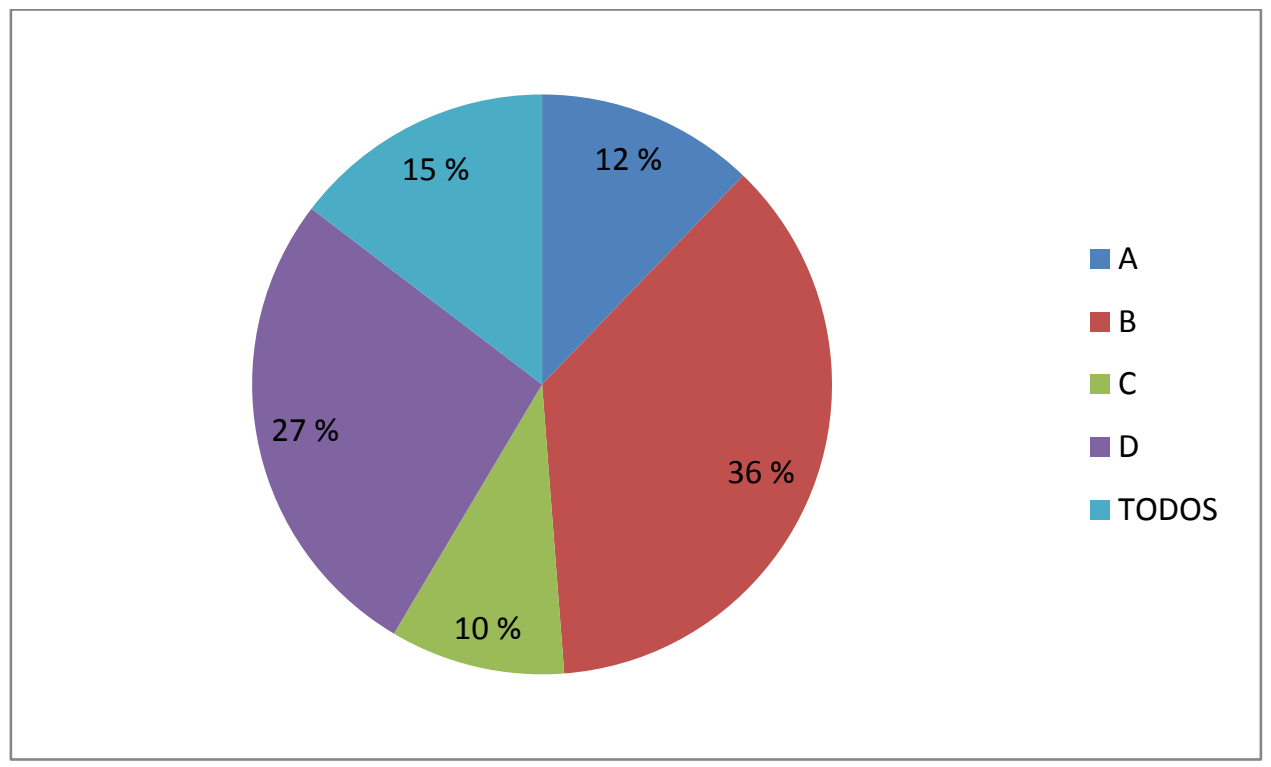

Al preguntar si el alumno cuenta con los medios necesarios para desarrollarse como empresario en el futuro, $54 \%$ contestó de forma afirmativa, mientras que $2 \%$ de los encuestados no contestó a la pregunta.

Figura 6. Se cuenta con medios necesarios para desarrollarse como empresario.

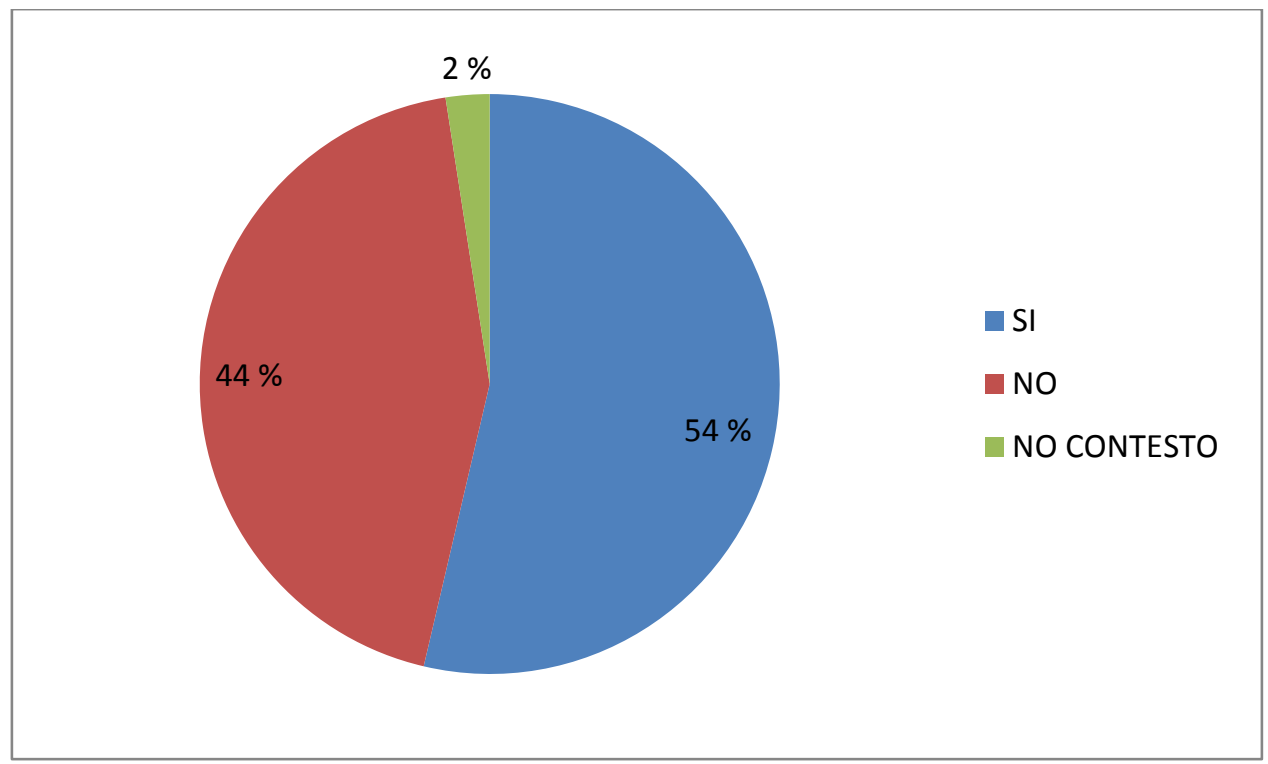

(Examen diagnóstico elaborado por el Comité de Acreditación MVZ 2016.

Reporte elaborado por la Maestra Adriana de la Rosa Figueroa, Coordinación de Carrera, 07 septiembre 2016.) 


\section{Discusión y relevancia}

\section{Concepto de perfil de ingreso}

Hoy en día, existen diversas definiciones sobre lo que es un perfil de ingreso. A continuación se muestran algunas de ellas.

- El diccionario de la Real Academia de la Lengua Española (2017) define la palabra ingreso, proveniente del latín ingressus, como el acto de ser admitido en una corporación o de empezar a gozar de un empleo o alguna otra cosa.

- La Secretaría de Educación Pública (2016) define el perfil de ingreso como el conjunto de conocimientos, habilidades, actitudes y valores que debe de reunir y demostrar el aspirante a cursar una licenciatura, con la finalidad de garantizar su formación al concluir sus estudios profesionales.

- La Universidad de Castilla - La Mancha (UCLM, 2017) maneja que un perfil de ingreso integra la descripción conceptual de las características deseables en el alumno de nuevo ingreso en términos de conocimientos, habilidades y actitudes favorables para cursar y terminar con mayores posibilidades de éxito los estudios que inicia. Además da cuenta de las opciones académicas cursadas, notas académicas obtenidas y datos de carácter sociológico de interés.

- En el manual de Perfiles de Ingreso / Egreso: Sistema de garantía interna de calidad de la Universidad Católica de San Antonio (2001), se define este concepto como la descripción conceptual de las características deseables en el alumno de nuevo ingreso en términos de conocimientos, habilidades y actitudes favorables para cursar y terminar con mayores posibilidades de éxito los estudios que inició. Además de contar con las opciones académicas cursadas, notas académicas obtenidas y datos de carácter sociológico de interés. 


\section{La importancia social del profesional en medicina veterinaria}

Podemos afirmar que el ejercicio profesional del médico veterinario y/o zootecnista puede estar asociado a una serie de competencias que deben ser logradas durante su formación académica:

$\checkmark$ Capacidad para diseñar y mejorar la efectividad de los sistemas de producción, procesamiento, industrialización, administración y comercialización de los recursos, bienes y servicios de las empresas agropecuarias o agroindustriales, así como nuestros recursos naturales y ambientales, aprovechando las bondades tecnológicas, modernas, disponibles en todos los campos de las ciencias de una manera sostenible.

$\checkmark$ Conocimiento de las técnicas para establecer diagnósticos acertados, instaurar tratamientos eficaces y de la misma manera, criterio para instaurar las medidas necesarias en la prevención de las enfermedades animales que se transmiten al humano.

$\checkmark$ Tener sólidos conocimientos científicos y técnicos en las distintas ramas de las ciencias pecuarias, dentro de una cultura investigativa, que les permitirá el manejo eficaz de las diversas alternativas viables de solución a la problemática sectorial.

$\checkmark$ Certificar el estado y la calidad de diversos productos de origen animal destinados al consumo o beneficio humano y lo relacionado con la salud pública.

$\checkmark$ Participar y liderar activamente las diversas labores que tienen que ver con las formas asociativas de producción, mercadeo e investigación sectorial que le permitan participar dinámicamente en ellas, con especial énfasis en la economía solidaria.

$\checkmark$ Conocer y asimilar la realidad económica y actual de nuestro entorno social, económico, productivo y administrativo, para entender la necesidad de renovación y participar activamente en equipos interdisciplinarios que contribuyan a la solución proactiva de los problemas y retos que se ofrecen dentro de la misma sociedad en la cual está inmerso.

$\checkmark$ Creatividad para incentivar la generación de nuevos servicios, productos, bienes, procesos, tratamientos y métodos relacionados con el sector agropecuario, que le permitan acrecentar o acceder a nuevos mercados. 
$\checkmark$ Formación investigativa que le permite acceder al conocimiento de las oportunidades de desempeño y desarrollo de las últimas tecnologías científicas, relacionadas con la medicina veterinaria y zootecnia.

Es indudable la importancia del rol desempeñado por el médico veterinario zootecnista en el desarrollo de nuestra sociedad, pero en la mayoría de los países sudamericanos esa importancia no siempre es reconocida y en ocasiones es ignorada. La preparación idónea y pertinente del MVZ es fundamental en el logro de su aceptación como profesional útil y necesario para la solución de los problemas que le conciernen en la sociedad. La preparación del MVZ depende fundamentalmente, por una parte, de la calidad de los conocimientos impartidos por las instituciones a lo largo de la formación académica y, por otra parte, del interés y motivación personal del estudiante.

\section{Ética médica y médica veterinaria}

La ética médica es una disciplina que se ocupa del estudio de los actos médicos desde el punto de vista moral y que los califica como buenos o malos, a condición de que ellos sean voluntarios y conscientes. Al decir "actos médicos" debe hacerse referencia a los que adelanta el profesional de la medicina o la medicina veterinaria en el desempeño de su profesión frente al paciente y la sociedad. Los aspectos que lleve a cabo el individuo en función de su vida privada, no profesional, caerán en el campo de la ética general, misma que permite juzgar los actos de cualquier persona (Trujillo Mascia, 2005).

El médico y el médico veterinario actúan en función profesional también en actividades distintas a la clínica y a la cirugía, como son aquellas relacionadas con la salud pública, el laboratorio clínico, la patología, la medicina legal, la investigación biológica, la reproducción, la gerencia, la extensión, la docencia, etcétera. Precisamente, uno de los defectos que tiene la ética tradicional es que en el juzgamiento moral del médico y del médico veterinario se redujo su campo de acción a lo que hagan al lado del paciente o en el quirófano (Trujillo Mascia, 2005).

En la actualidad se aceptan como tres los principios considerados leyes morales en ética médica y médica veterinaria. Estos principios son de carácter imperativo, racional, 
universal, categórico, auto-obligatorio y consciente: autonomía, beneficencia/nomaleficencia y justicia (Trujillo Mascia, 2005).

\section{Principio de Autonomía}

Hace referencia a la libertad que tiene una persona para establecer sus normas personales de conducta, basada en su propio sistema de valores y principios; es decir, expresa autorregulación, autovaloración y autogobierno, sin constricciones de ningún tipo. La persona autónoma determina por sí misma el curso de sus acciones de acuerdo a un plan escogido por ella misma. Por supuesto que durante el acto médico, la autonomía tiene que ver con la del paciente y no con la del médico, y en el caso de la medicina veterinaria tiene que ver con la autonomía del dueño del paciente. Hoy en día, la autonomía ha llegado a convertirse en la consigna que simboliza el derecho moral y legal de los pacientes y de los dueños de los pacientes, a adoptar sus propias decisiones sin restricción ni coerción, por más bienhechoras que sean las intenciones del médico o del médico veterinario. Al fin y al cabo sus decisiones autónomas responderán a su propia conciencia, a su propia ética (Trujillo Mascia, 2005).

\section{Principio de Beneficencia/No-Maleficencia}

La máxima latina primum non nocere (primero no hacer daño) siempre ha sido el fundamento de la moralidad en el ejercicio médico humano o veterinario. Se debe tomar en cuenta que no-maleficencia abarca no sólo la violencia y el daño que pueda ocasionarse, sino también el riesgo de daño. De ahí que para evitarlo se requiera que el médico y el médico veterinario estén cuidadosamente atentos. La ausencia de malicia o de intención nunca ampara de la violación del principio de no-maleficencia (Trujillo Mascia, 2005).

\section{Principio de Justicia}

Desde la perspectiva de la justicia distributiva, se acepta que no sólo la sociedad tiene la obligación moral de proveer o facilitar a todos los individuos y aún más a todos los organismos, animales o vegetales, un acceso igualitario al ambiente, a la ecología, a la salud y al respeto y tolerancia de la variabilidad, la asimetría y la desigualdad; sino que además todo individuo $\mathrm{u}$ organismo tiene el derecho universal inalienable de acceder a ellos. Por lo tanto, el médico y médico veterinario en ejercicio deberán, para actuar dentro 
del marco ético, estar familiarizados e identificados con los valores y principios morales que sustentan el sistema ético-médico (Trujillo Mascia, 2005).

\section{Consistencia de la formación de competencias en educación veterinaria}

Las instituciones mexicanas que imparten estudios en medicina veterinaria no han permanecido al margen de las iniciativas que promueven el enfoque de competencias; sin embargo, más allá de esta tendencia (de sí evidente en diversos aspectos relacionados con la calidad de la educación), está el hecho de que las competencias son inherentes al proceso formativo, independientemente del modelo educativo adoptado. En este sentido, la formación de competencias está fuera de toda disyuntiva, salvo la que representan dos escenarios alternativos en situaciones dadas: uno que favorece la adquisición y desarrollo de competencias académico-profesionales en el estudiantado; y otro donde las condiciones y circunstancias apartan al educando del aprendizaje necesario para alcanzar ese fin (Bautista Climent, 2016).

\section{Solidez de las competencias}

Independientemente del programa de estudios que se trate, el desarrollo de las competencias del médico veterinario, en atención a distintos factores (naturaleza, contextos, significados, intereses, dominios, mérito, complejidad, alcances, límites), se asocia a dos momentos referenciales: el ingreso y el egreso de los estudiantes de modo que la evolución de las competencias de los educandos, de un momento a otro, es evidencia, a su vez, de los logros del programa en cuestión, bajo condiciones dadas (Bautista Climent, 2016).

A continuación se muestra un cuadro comparativo con los diferentes perfiles de ingreso de las principales universidades que cuentan con el Programa Educativo de Medicina Veterinaria y Zootecnia. 


\begin{tabular}{|c|c|}
\hline UNIVERSIDAD & $\begin{array}{l}\text { PERFIL DE INGRESO A LA } \\
\text { LICENCIATURA DE MVZ }\end{array}$ \\
\hline $\begin{array}{l}\text { Universidad de Guadalajara } \\
\text { (U de G) } \\
\text { Centro Universitario de los Altos } \\
\text { (CUAltos) }\end{array}$ & $\begin{array}{l}\text { 1. Actitudes (comportamiento y } \\
\text { disposiciones). Características } \\
\text { particulares del desarrollo personal que } \\
\text { cada individuo ha aprehendido y } \\
\text { madurado como producto de su } \\
\text { experiencia de vida deben ser: iniciativa, } \\
\text { responsabilidad, autoaprendizaje, } \\
\text { disciplina, liderazgo, sociabilidad, } \\
\text { honestidad, ética, tenacidad, vocación de } \\
\text { servicio, afinidad e interés por los } \\
\text { fenómenos biológicos, disponibilidad para } \\
\text { el trabajo de laboratorio y de campo. } \\
\text { 2. Aptitudes yopacidades } \\
\text { habilidades). Disposición natural que la } \\
\text { persona posee o adquiere, son: saber } \\
\text { aprovechar los recursos disponibles; saber } \\
\text { dirigir; capacidad para administrar, realizar } \\
\text { trabajo en equipo y para el análisis crítico; } \\
\text { capacidad de síntesis y de abstracción; } \\
\text { pensamiento lógico matemático; habilidad en la } \\
\text { lectura de comprensión, en redacción y } \\
\text { composición, creatividad; habilidad en } \\
\text { comunicación oral y escrita. } \\
\text { 3. yntecedentes académicos. Son los estudios } \\
\text { formales realizados previamente. Haber } \\
\text { cursado el Bachillerato. }\end{array}$ \\
\hline $\begin{array}{l}\text { Universidad Nacional Autónoma de } \\
\text { México } \\
\text { (UNAM) }\end{array}$ & $\begin{array}{l}\text { Los aspirantes a ingresar al PUIMVZ deberán } \\
\text { reunir características esenciales en los } \\
\text { siguientes } \\
\text { Conocimientos } \\
\text { - Ser médicos veterinarios zootecnistas o } \\
\text { médicos veterinarios con deseos de } \\
\text { consolidar los conocimientos } \\
\text { profesionales y de desarrollar las } \\
\text { habilidades clínicas o zootécnicas que } \\
\text { les permita aplicar una metodología } \\
\text { enfocada a la solución de problemas. } \\
\text { Cualidades personales } \\
\text { Disciplina, actitud crítica y } \\
\text { reflexiva, proactividad, comunicación } \\
\text { eficaz y deseos de brindar un mejor } \\
\text { servicio, sustentado en valores, calidad } \\
\text { y eficiencia. }\end{array}$ \\
\hline
\end{tabular}




\begin{tabular}{|c|c|}
\hline & $\begin{array}{l}\text { Habilidades } \\
\text { - Competencias básicas de medicina o } \\
\text { zootecnia en el campo de conocimiento } \\
\text { de interés. } \\
\text { Permanencia } \\
\text { Obtener calificaciones aprobatorias en } \\
\text { todos los módulos. } \\
\text { Cumplir con la totalidad de las } \\
\text { actividades previstas en cada módulo, } \\
\text { que incluyen revisión biblio- } \\
\text { hemerográfica, guardias (nocturnas y } \\
\text { de fin de semana) y presentación de } \\
\text { seminarios. Así como estudio de casos } \\
\text { clínicos, discusión de casos clínicos, } \\
\text { práctica clínica, o bien, evaluación y } \\
\text { resolución de problemáticas de manejo, } \\
\text { genéticas, } \\
\text { administrativas o nutricionales. } \\
\text { Apegarse a los reglamentos internos de } \\
\text { la Facultad y del departamento o centro } \\
\text { que coordine el campo de conocimiento } \\
\text { involucrado. }\end{array}$ \\
\hline $\begin{array}{l}\text { Universidad Autónoma de Querétaro } \\
\text { (UAQ) } \\
\text { Facultad de Ciencias Naturales }\end{array}$ & $\begin{array}{l}\text { El aspirante a cursar la Licenciatura en Medicina } \\
\text { Veterinaria y Zootecnia deberá: } \\
\text { Tener valores, hábitos de estudio y disposición por } \\
\text { el trabajo en equipo. } \\
\text { Mostrar competencias de análisis y síntesis. } \\
\text { Estar interesado en el conocimiento de la } \\
\text { problemática ecológica y dispuesto a mejorar su } \\
\text { entorno. } \\
\text { Mostrar disposición a trabajar con animales. } \\
\text { Tener conocimientos en el Área Químico-Biológicas, } \\
\text { en inglés y técnicas computacionales. }\end{array}$ \\
\hline $\begin{array}{l}\text { Universidad Autónoma de Nuevo } \\
\text { León } \\
\text { (UANL) } \\
\text { Facultad de Medicina Veterinaria y } \\
\text { Zootecnia }\end{array}$ & $\begin{array}{l}\text { Los estudiantes deberán tener los siguientes } \\
\text { conocimientos: español, sus orígenes y aplicación; } \\
\text { matemáticas; biología de los seres vivos y } \\
\text { especialmente del ser humano; historia universal, de } \\
\text { México y de Nuevo León; metodología de la } \\
\text { investigación; filosofía, desarrollo y aprendizaje } \\
\text { humano; física; química; computación; inglés; } \\
\text { avances científicos actuales; ética y derechos }\end{array}$ \\
\hline
\end{tabular}




\begin{tabular}{|c|c|}
\hline & $\begin{array}{l}\text { humanos; conceptos de derecho; elementos } \\
\text { relacionados con la economía y sus problemas en } \\
\text { nuestro país; el medio ambiente y su preservación; y } \\
\text { el hombre y su sociedad. Además, los estudiantes } \\
\text { aspirantes a ingresar a la carrera de Médico } \\
\text { Veterinario Zootecnista deberán reunir los siguientes } \\
\text { atributos: CONOCIMIENTOS: básicos de biología, } \\
\text { química, física, geografía, matemáticas, probabilidad } \\
\text { y estadística, inglés, metodología de la ciencia, } \\
\text { gramática, ortografía y redacción, así como manejo } \\
\text { básico de computación. HABILIDADES: capacidad } \\
\text { para expresar sus pensamientos de manera } \\
\text { ordenada y coherente, a través del lenguaje oral y } \\
\text { escrito, redacción adecuada en español, lectura de } \\
\text { comprensión del español e inglés, uso elemental de } \\
\text { equipo y programa de cómputo, razonamiento } \\
\text { matemático. ACTITUDES: interés por la problemática } \\
\text { ambiental y productiva del país, y en particular en el } \\
\text { estado de Nuevo León, interés por el estudio de los } \\
\text { recursos naturales y/o sistemas de producción } \\
\text { agropecuarios, capacidad de observación y } \\
\text { disposición para el trabajo de campo y laboratorio, } \\
\text { disposición para trabajo en equipo, disposición para } \\
\text { desarrollar su capacidad de auto-aprendizaje. } \\
\text { VALORES: responsabilidad, honradez, tolerancia, } \\
\text { puntualidad. }\end{array}$ \\
\hline $\begin{array}{l}\text { Universidad Autónoma del Estado de } \\
\text { México } \\
\text { (UAEM) } \\
\text { Facultad de Medicina Veterinaria y } \\
\text { Zootecnia }\end{array}$ & $\begin{array}{l}\text { VALORES: integridad, ética, moral, honestidad, } \\
\text { disciplina y un alto espíritu de responsabilidad. } \\
\text { INTERESES: académicos, científicos y humanistas; } \\
\text { así como disposición para el aprendizaje, } \\
\text { capacitación en métodos y técnicas en salud animal } \\
\text { y zootecnia. APTITUDES: de razonamiento lógico } \\
\text { con exactitud y rapidez en las respuestas; capacidad } \\
\text { de comunicación (tanto oral como escrita), capacidad } \\
\text { de observación, análisis y síntesis; comprensión de } \\
\text { problemáticas complejas y su solución constructiva; } \\
\text { capacidad de adaptabilidad ante retos inesperados, } \\
\text { creatividad e innovación; con un amplia visión de la } \\
\text { realidad, pensamiento positivo y entusiasmo; } \\
\text { disciplina en el trabajo y capacidad para la } \\
\text { autoadministración. ACTITUDES: capacidad para } \\
\text { integrarse y desarrollarse adecuadamente en } \\
\text { equipos de trabajo; reconocimiento multidisciplinario } \\
\text { de la vida comunitaria, actitud crítica y constructiva } \\
\text { ante problemáticas prácticas; constituirse en agente } \\
\text { de cambio, sensibilidad para reconocer los } \\
\text { problemas ambientales e intervenir en su solución; } \\
\text { capacidad para acceder a las fuentes de información } \\
\text { de manera rápida y eficaz. CONOCIMIENTOS: } \\
\text { dominio en áreas del nivel medio superior (biología, } \\
\text { anatomía, fisiología, matemáticas, investigación } \\
\text { documental, redacción, informática e inglés). }\end{array}$ \\
\hline $\begin{array}{l}\text { Universidad del Valle de México } \\
\text { (UVM) }\end{array}$ & $\begin{array}{l}\text { Conocimientos: } \\
\text { - Conceptos básicos de biología. } \\
\text { - Conceptos básicos de química. } \\
\text { - Conceptos básicos de física } \\
\text { - Conceptos básicos de fisiología } \\
\text { - Manejo de sistemas de información y } \\
\text { - Nomputación } \\
\text { Habilidades: } \\
\text { - Capacidad de análisis y síntesis de la }\end{array}$ \\
\hline
\end{tabular}




\begin{tabular}{|c|c|}
\hline & 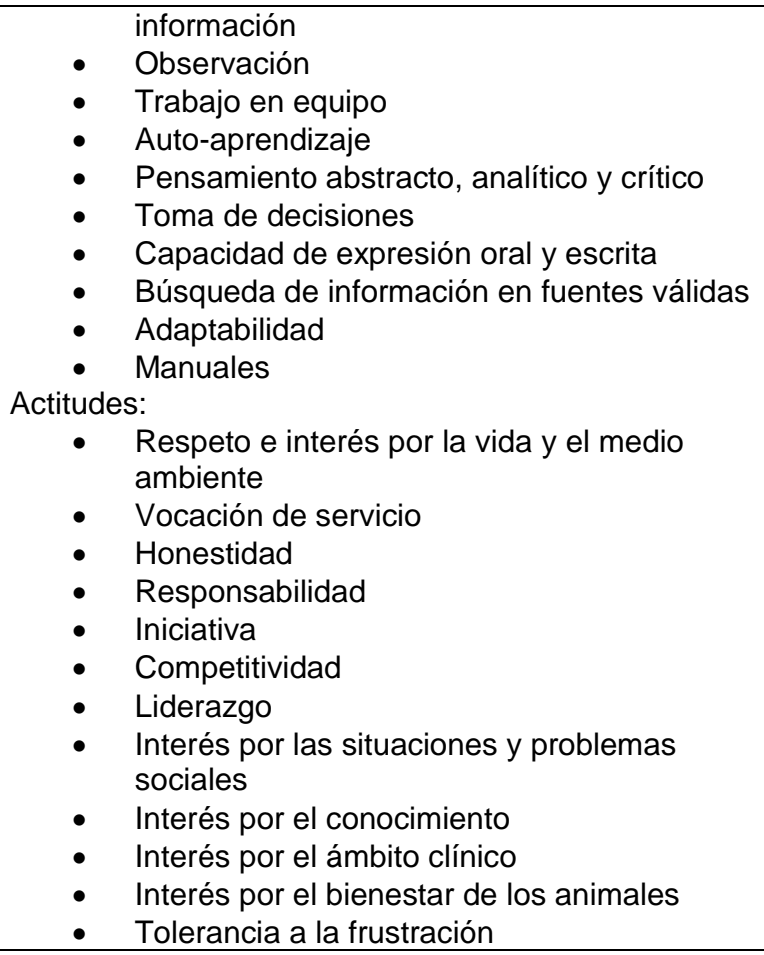 \\
\hline Universidad de La Salle & $\begin{array}{l}\text { Intereses } \\
\text { Gusto por las matemáticas básicas para análisis } \\
\text { bioestadísticos, desarrollo de negocios y estudios de } \\
\text { genética. } \\
\text { Por la biología o la vida en general } \\
\text { Por la naturaleza } \\
\text { En el trato con animales } \\
\text { Actitud de servicio } \\
\text { Habilidades } \\
\text { Razonamiento abstracto } \\
\text { Análisis y síntesis de la información y datos } \\
\text { Organización de la información y del trabajo } \\
\text { Aplicación de la creatividad } \\
\text { Trabajo en equipo } \\
\text { Toma de decisiones } \\
\text { Valores } \\
\text { Servicio } \\
\text { Respeto por la vida y la naturaleza } \\
\text { Responsabilidad } \\
\text { Disciplina } \\
\text { Honestidad }\end{array}$ \\
\hline $\begin{array}{l}\text { Universidad Autónoma de Ciudad } \\
\text { Juárez } \\
\text { (UACJ) }\end{array}$ & $\begin{array}{l}\text { El estudiante que ingrese al programa de Medicina } \\
\text { Veterinaria y Zootecnia deberá tener conocimientos } \\
\text { del lenguaje oral y escrito; capacidad de retención y } \\
\text { análisis crítico de los conocimientos, disponibilidad } \\
\text { de trabajo en equipo. Aunado a esto deberá mostrar } \\
\text { interés por el cuidado de los animales, los problemas } \\
\text { pecuarios, de salud pública y deberá contar con una } \\
\text { preparación durante los estudios de preparatoria } \\
\text { que le permitan tener conocimientos básicos sobre } \\
\text { biología, química, anatomía e inglés a nivel de }\end{array}$ \\
\hline
\end{tabular}




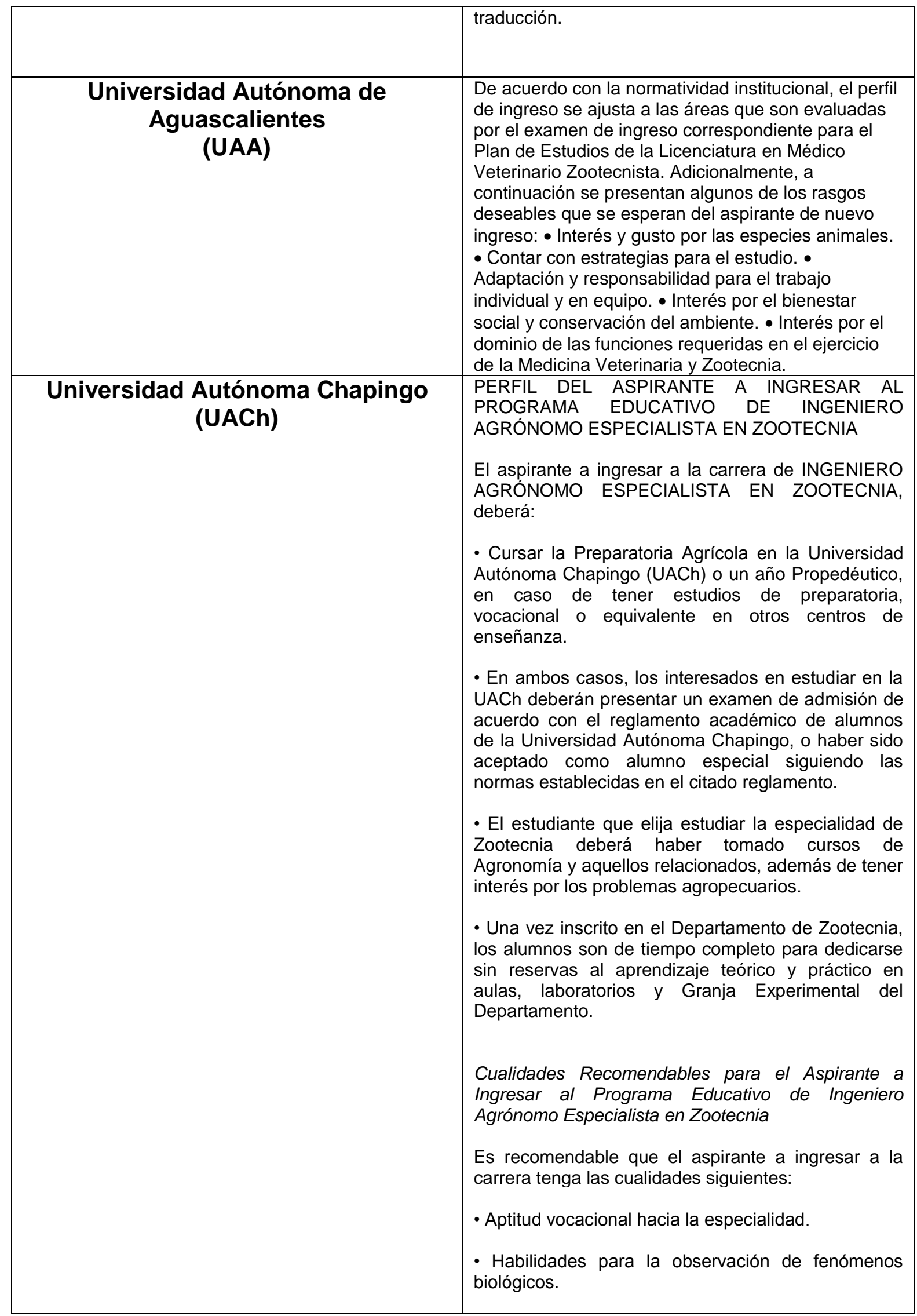




\begin{tabular}{|c|c|}
\hline & $\begin{array}{l}\text { - Disciplina y hábito de estudio, así como motivación } \\
\text { hacia el estudio dentro y fuera del aula. } \\
\text { - Buena salud y capacidad de adaptación a trabajo } \\
\text { en ambientes libres. } \\
\text { - Resistencia a trabajos prolongados en el campo. } \\
\text { - Habilidades para relacionarse con los campesinos y } \\
\text { personal de nivel socio-económico diverso. } \\
\text { - Auténtico interés por la optimización de los } \\
\text { recursos agropecuarios y mejoramiento social, } \\
\text { cultural y económico de los habitantes de zonas } \\
\text { rurales. } \\
\text { - Responsabilidad y actitud propositiva para el } \\
\text { trabajo individual y colectivo dentro y fuera del } \\
\text { Departamento. }\end{array}$ \\
\hline $\begin{array}{c}\text { Instituto Tecnológico de Sonora } \\
\text { (ITSON) }\end{array}$ & $\begin{array}{l}\text { El aspirante a ingresar a la carrera de Medicina } \\
\text { Veterinaria y Zootecnia deberá contar con: } \\
\text { - } \quad \text { Estudios de nivel medio superior con orientación } \\
\text { al área médico- biológica, químico-biológico, } \\
\text { ciencias naturales o equivalente. } \\
\text { - Vocación para el trato y manejo humanitario de } \\
\text { los animales. } \\
\text { - Disposición para el trabajo al aire libre (en } \\
\text { campo). } \\
\text { - Compromiso para el cuidado del medio } \\
\text { - Conbiente y la biodiversidad. } \\
\text { - Conivel de aplicación. } \\
\text { - Conocimientos y habilidades en estadística a } \\
\text { nivel de aplicación. } \\
\text { - Conocimientos de química inorgánica y orgánica } \\
\text { - Habilidades lingüínsticas. } \\
\text { - Conocimientos básicos de computación. }\end{array}$ \\
\hline $\begin{array}{l}\text { Universidad Autónoma de Zacatecas } \\
\text { (UAZ) }\end{array}$ & $\begin{array}{l}\text { Los aspirantes a la licenciatura de Medicina } \\
\text { Veterinaria y Zootecnia de la Universidad Autónoma } \\
\text { de Zacatecas, acreditarán estudios de bachillerato en } \\
\text { Ciencias Biológicas o Bachillerato Único. } \\
\text { El aspirante ha de tener una comprensión clara de su } \\
\text { entorno: de la naturaleza y de la sociedad, de la } \\
\text { forma en que una y otra se conforman y se } \\
\text { comportan, de la manera como la experiencia } \\
\text { humana sobre ellas se ha ido sistematizando. Esto } \\
\text { implica conocimientos conceptuales, } \\
\text { procedimentales y actitudinales. } \\
\text { Debe dominar la lengua materna y tener nociones de } \\
\text { otras lenguas (inglés, francés o alemán) y hacer uso } \\
\text { de paquetería de cómputo. } \\
\text { Dentro de las habilidades que el aspirante debe } \\
\text { poseer, se encuentran la capacidad para buscar y } \\
\text { localizar información, seleccionarla, probarla, } \\
\text { ordenarla, valorarla, y utilizarla oportunamente; para } \\
\text { identificar el punto problemático en una situación } \\
\text { dada y para plantear problemas y distinguir y } \\
\text { localizar variables. } \\
\text { En el plano actitudinal son indispensables: la }\end{array}$ \\
\hline
\end{tabular}




\begin{tabular}{|c|c|}
\hline & $\begin{array}{l}\text { inquietud intelectual, la imaginación creativa, la } \\
\text { solidaridad y la disciplina, Valores: responsabilidad, } \\
\text { justicia, honestidad, pensamiento crítico- científico, } \\
\text { honradez y compromiso social. } \\
\text { Bachillerato terminado exclusivo de Ciencias } \\
\text { Biológicas. }\end{array}$ \\
\hline $\begin{array}{l}\text { Universidad Veracruzana } \\
\text { (UV) }\end{array}$ & $\begin{array}{l}\text { Respecto al perfil de ingreso algunas } \\
\text { recomendaciones acerca de las habilidades } \\
\text { intelectuales, intereses personales y aspectos de } \\
\text { personalidad deseables para la carrera de } \\
\text { veterinaria, son: } \\
\text { - Capacidad de análisis y síntesis, razonamiento } \\
\text { lógico, capacidad de creación e innovación, memoria } \\
\text { visual y auditiva, adaptación a las nuevas } \\
\text { tecnologías, capacidad de adaptación a equipos de } \\
\text { trabajo. } \\
\text { - Interés por la investigación, la biología, la salud, la } \\
\text { ecología, el mundo natural y en especial por los } \\
\text { animales. } \\
\text { - Habilidades para combinar el trabajo individual y el } \\
\text { trabajo en equipo, sensibilidad y paciencia con los } \\
\text { animales. }\end{array}$ \\
\hline $\begin{array}{l}\text { Universidad Juárez Autónoma de } \\
\text { Tabasco } \\
\text { (UJAT) }\end{array}$ & $\begin{array}{l}\text { Es recomendable que el aspirante posea las } \\
\text { siguientes características, conocimientos y } \\
\text { habilidades: } \\
\text { * Haber cursado el bachillerato en el área de las } \\
\text { Ciencias Químico Biológica. } \\
\text { * Conocimientos elementales de computación e } \\
\text { inglés. } \\
\text { * Interés por los animales, el medio rural y sus } \\
\text { actividades, así como la conservación del medio } \\
\text { ambiente. } \\
\text { * Habilidades para la expresión oral y escrita. } \\
\text { * Capacidad para integrarse a grupos de trabajo. } \\
\text { * Que posea ingenio y creatividad. } \\
\text { * Responsabilidad y disciplina. }\end{array}$ \\
\hline $\begin{array}{l}\text { Universidad Autónoma de Nayarit } \\
\text { (UAN) }\end{array}$ & $\begin{array}{l}\text { El estudiante que desee ingresar a la carrera de } \\
\text { Medicina Veterinaria y Zootecnia deberá tener: } \\
\text { 1. Un concepto definido de la actividad del } \\
\text { médico veterinario en el área pecuaria, } \\
\text { como fuente de producción de alimentos, } \\
\text { así como en las áreas de la medicina } \\
\text { veterinaria y salud pública. } \\
\text { 2. Interés en la solución de problemas de } \\
\text { salud y producción animal. } \\
\text { 3. Buen manejo del lenguaje oral y escrito. } \\
\text { 4. Disponibilidad para el trabajo en equipo. } \\
\text { 5. Actitud favorable hacia el aprendizaje } \\
\text { Asimismo, es deseable que tenga: } \\
\text { 1. Bases firmes de química y biología. } \\
\text { 2. Dominio básico del idioma inglés, a nivel de } \\
\text { comprensión de lectura y traducción escrita. } \\
\text { 3. Capacidad de retención y análisis crítico de } \\
\text { los conocimientos. } \\
\text { 4. Manejo de equipo de cómputo. } \\
\text { 5. Respeto por el medio ambiente. }\end{array}$ \\
\hline
\end{tabular}




\section{Conclusión}

Las diferentes universidades en las que se imparte la licenciatura de medicina veterinaria coinciden en muchos aspectos en su perfil de ingreso; sin embargo, son pocos los estudiantes que conocen el perfil de ingreso a su universidad. Además, existen varios aspectos que el estudiante debería cubrir y tomar en cuenta que tiene que contar con aptitudes tales como: capacidad de trabajar en equipo, tener una actitud favorable hacia el aprendizaje permanente, interés por los animales, el medio rural y sus actividades, así como la conservación del medio ambiente.

El aspirante debe tener una comprensión clara del entorno: de la naturaleza y de la sociedad, de la forma en que una y otra se conforman y se comportan, de la manera como la experiencia humana sobre ellas se ha ido sistematizando.

La gran mayoría de los alumnos que ingresan a la licenciatura de medicina veterinaria y zootecnia no cuenta con los recursos necesarios para trabajar independientemente; sin embargo, aspira a una buena educación para en algún momento poder contar con esos recursos y obtener la manera de contar con su propio medio de trabajo, sea una granja, una clínica o algún negocio donde sea indispensable tener los conocimientos adquiridos durante su preparación académica. 


\section{Bibliografía}

Bautista Climent, J. (2016). Un análisis sobre la consistencia de la formación de competencias en Educación Veterinaria. (2016). UAM. México.

Benemérita Universidad de Guadalajara (2016). Perfil de ingreso. 16/dic/2016, de Centro Universitario de los Altos Sitio web: http://www.cualtos.udg.mx/ofertaacademica/licenciatura-medicina-veterinaria-zootecnia/perfil-ingreso

Educación veterinaria en México (2015). Prospectiva de la Facultad de Medicina Veterinaria y Zootecnia de la UNAM. México.

Instituto Tecnológico de Sonora (ITSON) (2016). Perfil de ingreso. 17/dic/2016. Sitio web: http://www.itson.mx/oferta/mvz/Paginas/mvz.aspx

Manual de Perfiles de Ingreso / Egreso: Sistema de garantía interna de calidad (2011). 15/feb/2017. Universidad Católica de San Antonio (UCSA). España. Sitio web: http://www.ucam.edu/sites/default/files/Mayo_13/pcl-02-perfiles-de-ingreso.pdf

Secretaría de Educación Pública (2017). Perfil de ingreso en la Educación Normal. 14/feb/2017.

Sitio

web: http://www.dgespe.sep.gob.mx/reforma_curricular/planes/lepri/plan_de_estudios/perf il_ingreso.

Serrano Novoa, C. A. et al. (2008). La importancia social del profesional en Medicina Veterinaria. RedVet. 1695-7504

Trujillo Mascia, N. (2005). Ética Médico Veterinaria y Deontología de la DCV-UCLA. EEUU.

Universidad Autónoma de Aguascalientes (UAA) (2016). Perfil de ingreso. 18/dic/2016. México. Sitio web: http://www.uaa.mx/direcciones/dgdp/catalogo/ciencias_agropecuarias/medico_veteri nario_zootecnista.pdf

Universidad Autónoma de Ciudad Juárez (2016). Perfil de ingreso. 18/dic/2016. México. Sitio web: http://www.uacj.mx/ICB/DCV/LMVZ/Paginas/default.aspx 
Universidad Autónoma de Chapingo (UACh) (2016). Perfil de ingreso. México. 17/dic/2016. Sitio web: http://zootecnia.chapingo.mx/perfil-del-aspirante.html

Universidad Autónoma del Estado de México (UAEM) (2016). Perfil de ingreso. 17/dic/2016. México. Sitio web: http://veterinaria.uaemex.mx/_docs/37_54_Plan\%20de\%20estudios.pdf

Universidad Autónoma de México (UNAM) (2016). Perfil de ingreso. 16/dic/2016. México. Sitio web: http://www.fmvz.unam.mx/fmvz/sua/internado/perfil.html . 16/dic/2016.

Universidad Autónoma de Nuevo León (UANL) (2016). Perfil de ingreso. 17/dic/2016. México. Sitio web: http://www.veterinaria.uanl.mx/plan-estudios.html

Universidad Autónoma de Querétaro (UAQ) (2016), Perfil de ingreso. 16/dic/2016. México. Sitio web: http://www.uaq.mx/index.php/oferta-educativa/programaseducativos/fcn/licenciaturas-fcn/licenciatura-en-medicina-veterinaria-y-zootecnia .

Universidad Autónoma de Nayarit (UAN) (2016). Perfil de ingreso. 17/dic/2016. México. Sitio web: http://www.uan.edu.mx/es/medicina-veterinaria-y-zootecnia

Universidad Autónoma de Zacatecas (UAZ) (2016). Perfil de ingreso. 17/dic/2016. México. Sitio web: http://nautilus.uaz.edu.mx/ofertaeducativa/view/ofertaEducativa.php?idPrograma=15 $\underline{3020 \& \text { idUnidad }=22000}$

Universidad de Castilla La Mancha (UCLM) (2017). 14/feb/2017. España. Sitio web: www.uclm.es

Universidad del Valle de México (UVM) (2016). Perfil de ingreso. 17/dic/2016. México. Sitio web: http://www.universidaduvm.mx/

Universidad La Salle. (2016). Perfil de ingreso. 17/dic/2016. México. Sitio web: http://bajio.delasalle.edu.mx/oferta/oferta1.php? $=1 \& p=8$

Universidad Juárez Autónoma de Tabasco (UJAT) (2016). Perfil de ingreso. 17/dic/2016. México. Sitio web: 
http://www.archivos.ujat.mx/2011/div_daca/lic_MVZ/5\%20Perfil\%20de\%20Ingreso. pdf

Universidad Veracruzana (UV) (2016). Perfil de ingreso. 17/dic/2016. México. Sitio web: https://www.uv.mx/veracruz/fmvz/oferta-educativa/mvz/ 